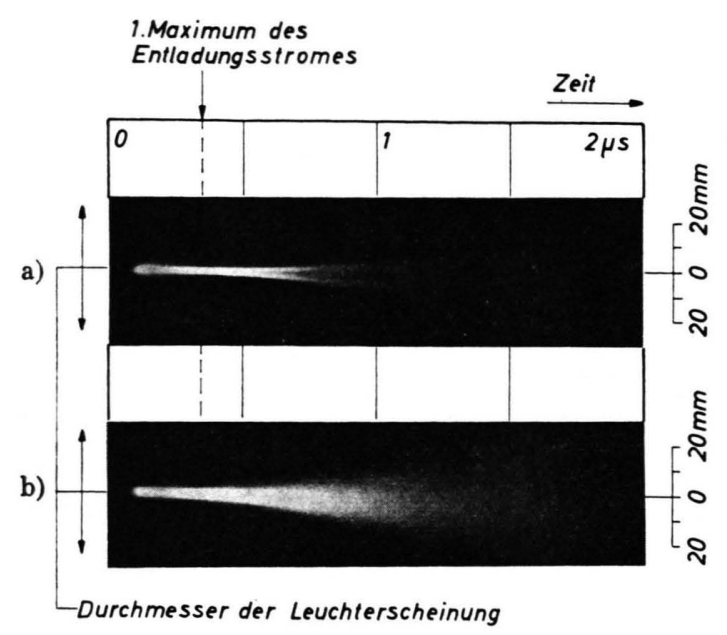

Abb. 4. Schmierbildaufnahmen des Plasmaquerschnitts

a) in $60 \mathrm{~mm}$ Abstand von der Masse-Elektrode,

b) in $180 \mathrm{~mm}$ Abstand von der Masse-Elektrode.

Die Messungen wurden mit einem STL-Bildwandler ausgeführt. Für die Aufnahmen wurde ein $4,5 \mathrm{~mm}$ hoher Querschnitt der Plasmasäule auf die Photokathode des Bildwandlers abgebildet. Die zeitliche Zuordnung von Entladungsstrom und Bildwandleraufnahme ist mit einer Ungenauigkeit von ungefähr \pm 50 nsec behaftet.
Bildwandleraufnahmen, bei denen die Zeitabhängigkeit der Leuchtdichteverteilung eines Plasmaquerschnitts nach der üblichen Schmierbildtechnik aufgenommen wurde, zeigen, daß sich die radiale Struktur der Leuchtdichte längs der Plasmasäule ändert. In der Nähe der Masse-Elektrode ist der Kern der Plasmasäule deutlich dunkler als der Rand (Abb. 4 a). In größerem Abstand von der Masse-Elektrode ist keine ausgeprägte radiale Abhängigkeit der Leuchtdichte wahrnehmbar (Abb. 4 b). Der Effekt kann qualitativ mit der leichten Divergenz des cluster-Strahls und der nur an einem Ende erfolgenden Injektion von Elektronen zur Zündung der Entladung erklärt werden.

Herrn Professor Dr. E. W. Becker danken wir für das Interesse, das er der Arbeit entgegenbrachte, Herrn Dr. O.F. Hagena für die Mitwirkung bei der Konstruktion der Vakuumapparatur und des Hochdruckkryostaten und Fräulein J. Riemenschneider für ihre Hilfe bei den Experimenten.

\title{
Paramagnetische Elektronenresonanz thermisch und durch Strahlung erzeugter Zentren in Bornitrid
}

\author{
G. Römelt
}

Institut für Angewandte Physik der Technischen Hochschule Clausthal

(Z. Naturforschg. 21 a, 1970-1975 [1966] ; eingegangen am 30. August 1966)

By X-rays, ultraviolet, and $\gamma$-radiation or by heating to more than $1850{ }^{\circ} \mathrm{C}$ in powdered or sintered boronnitride centres are produced, which give an EPR-spectrum with 10 lines $a=7.8 \pm 0.1$ Gauss apart with $g=2.0027 \pm 0.0003$ for $\mathrm{BN}$ of natural isotopic composition $\left(81.17 \%{ }^{11} \mathrm{~B} ; 18.83 \%{ }^{10} \mathrm{~B}\right)$, and a single line for ${ }^{10} \mathrm{BN}$ enriched to $91 \%{ }^{10} \mathrm{~B}$. The spectra are analysed as built up from 4 systems of spectra, caused by surroundings of 3 boron atoms, $3,2,1$, or 0 of which are ${ }^{11} \mathrm{~B}$, each occuring with the proper probability. The intensity of the different systems was calculated by assuming Gaussian line shape and equal $|\psi(0)|^{2}$ and line width for ${ }^{11} \mathrm{~B}$ and ${ }^{10} \mathrm{~B}$. It is possible, that the EPR is caused by impurity atoms on the places of $\mathrm{N}$ or in the centre of $\mathrm{B}_{3} \mathrm{~N}_{3}$-rings. Other spectra caused in $\mathrm{BN}$ by X-rays or by neutrons in a pile are discussed elsewhere ${ }^{3,8}$.

Kommerzielles Bornitrid liefert bei der paramagnetischen Elektronenresonanz (EPR) häufig ein 10-Linien-Spektrum (Abb. 1), als dessen Ursache die Hyperfeinwechselwirkung eines Elektrons mit den Kernspins von drei Atomkernen ${ }^{11} \mathrm{~B}$ vermutet wurde $^{1}$, wie sie z. B. in $53,5 \%$ der $\mathrm{B}_{3} \mathrm{~N}_{3}$-Ringe des $\mathrm{BN}$ vorliegen wegen der natürlichen Isotopenhäufigkeit $81,17 \%{ }^{11} \mathrm{~B}$ und $18,83 \%{ }^{10} \mathrm{~B} .{ }^{11} \mathrm{~B}$ hat den Kernspin $I=3 / 2$. Bei dreimaliger $(2 I+1)$-facher Niveau-

1 D. Geist u. G. Römelt, Solid State Commun. 2, 149 [1964]. aufspaltung und $\Delta m_{\mathrm{I}}=0$ bei der EPR ergeben sich zehn Linien.

In Abschnitt I wird eine Analyse des Spektrums durchgeführt, welche die Überlagerung der Einzellinien sowie den Beitrag der ${ }^{10} \mathrm{~B}$ mit $I=3$ berücksichtigt. Unter den Voraussetzungen von Abschnitt I läßt sich dann voraussagen, wie das EPR-Spektrum bei anderer Isotopenzusammensetzung aussehen müßte, z. B. für $91 \%{ }^{10} \mathrm{~B}$, das auch experimentell untersucht wurde. 
In Abschnitt II wird die Präparation von $\mathrm{BN}$ beschrieben, in III geklärt, wie $\mathrm{BN}$ thermisch behandelt werden muß, damit EPR-wirksame Zentren entstehen, also das 10-Linien-Spektrum auftritt. Diese Frage stellte sich, da bei verschiedenen Sorten von handelsüblichem BN das Spektrum unterschiedlich stark gefunden wurde.

In Abschnitt IV wird die EPR von bestrahltem $\mathrm{BN}$ behandelt, in $\mathrm{V}$ die von Kohlenstoff-dotiertem.

Abschnitt VI diskutiert die EPR-Zentren des BN im Zusammenhang mit seiner Kristallstruktur und grenzt sie gegen die freien aromatischen Radikale ab.

\section{Analyse des EPR-Spektrums von BN und ${ }^{10} \mathrm{BN}$}

Die EPR wurde an 6 Sorten BN-Pulver und 5 Sorten gesintertem $\mathrm{BN}$ verschiedener Hersteller sowie an je etwa 10 Sorten selbst hergestelltem BN- und ${ }^{10} \mathrm{BN}$-Pulver untersucht ${ }^{*}$. Die gewonnenen 10-LinienSpektren von natürlichem BN unterscheiden sich nicht nur nach der Intensität, sondern sie sind auch verschieden gut aufgelöst, z. Tl. von breiten Linien überlagert usw. Im folgenden sollen nur gut aufgelöste Spektren betrachtet werden, wie z. B. das der Abb. 1.

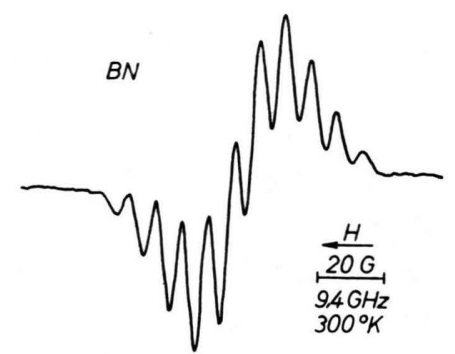

Abb. 1. EPR von grobkristallinem BN. $120 \mathrm{mg}$ Pulver S II, Korngröße bis $100 \mu$. Wie vom Hersteller bezogen. Modulation 0,7 Gauß Spitze-Spitze, Verstärkung 200.

Bei Kontakt-Hyperfeinwechselwirkung ist nach Fermi $^{2}$ der Abstand der Hyperfeinstrukturlinien

$$
a=\frac{8 \pi}{3} \frac{\mu}{I}|\psi(0)|^{2}
$$

abhängig vom Verhältnis Kernmoment $\mu$ zu Kernspin $I$ und von der Aufenthaltswahrscheinlichkeit

\footnotetext{
* Für die Herstellung eines Sinterstabes aus ${ }^{10} \mathrm{BN}$ mit $91 \%$ ${ }^{10} \mathrm{~B}$ danke ich Herrn Dr. LipP vom Elektroschmelzwerk Kempten.

2 E. Fermi, Z. Phys. 60, 320 [1930].
}

$|\psi(0)|^{2}$ des Elektrons am Kernort.

$$
\begin{array}{llll}
\text { Für }{ }^{11} \mathrm{~B} \text { ist } & I=3 / 2 ; & \mu=2,69 ; & \mu / I=1,8, \\
\text { für }{ }^{10} \mathrm{~B} & I=3 ; & \mu=1,80 ; & \mu / I=0,6 .
\end{array}
$$

Bei Kontaktwechselwirkung eines Elektrons mit drei Kernen ${ }^{11} \mathrm{~B}$ sollte das EPR-Spektrum aus 10 Linien der Intensitäten $1: 3: 6: 10: 12: 12: 10: 6: 3: 1$ bestehen. Das ist in Abb. 1 offensichtlich nicht erfüllt, weil

a) die Linienbreite so groß ist, daß die Linien sich gegenseitig überlagern und

b) die $18,83 \%{ }^{10} \mathrm{~B}$ ebenfalls zur EPR beitragen.

Bei geeigneter Wahl der Linienbreite läßt sich das Spektrum der Abb. 1 unter folgenden Annahmen näherungsweise berechnen:

a) Ursache des Spektrums ist die Kontakthyperfeinwechselwirkung eines nicht abgesättigten Elektrons mit drei Bor-Atomkernen (vgl. VI). Die vorhandene geringe anisotrope Wechselwirkung kann hier außer Betracht bleiben.

Damit Kontakthyperfeinwechselwirkung auftritt, muß das betreffende Elektron wenigstens zum Teil im sbzw. $\sigma$-Zustand vorliegen. Sie führt zu isotroper Hyperfeinaufspaltung, wie sie im BN offenbar auftritt: andernfalls könnten die 10 Linien nicht so gut getrennt sein, da die Untersuchungen nur an Pulver und polykristallinen Sinterkörpern durchgeführt werden konnten.

Eine geringfügige Anisotropie des 10-Linien-Spektruns, die auf die Beteiligung einer anisotropen Wechselwirkung hinweist, findet man bei gesintertem $\mathrm{BN}$ : Steht das Magnetfeld $H$ senkrecht auf der Achse des Sinterstabes, so sind die Linien wesentlich besser aufgelöst als für $H$ parallel zur Achse. Die Kriställchen des BN lagern sich beim Pressen offenbar bevorzugt so, daß die $c$-Achse mit der Stabachse zusammenfällt, was auch zu einer schwach ausgeprägten Textur von Debye-Scherrer-Aufnahmen führt ${ }^{3}$.

Einen $\sigma$-Anteil von $55 \%$ am Boratom ergeben auch die Kernresonanzuntersuchungen von SiLver und BRAY ${ }^{4}$ am BN.

b) $|\psi(0)|^{2}$ ist gleich groß an den Kernen ${ }^{11} \mathrm{~B}$ und ${ }^{10} \mathrm{~B}$. Wegen der Verhältnisse $\mu / I$ ist der Abstand $a$ der EPR-Linien von ${ }^{11} \mathrm{BN}$ dann gerade dreimal so groß wie der von ${ }^{10} \mathrm{BN}$.

c) Die Halbwertsbreite der Einzellinien $2 h \cdot \sqrt{2 \ln 2}$ ist gleich groß bei Beteiligung von ${ }^{11} \mathrm{~B}$ - oder ${ }^{10} \mathrm{~B}$ Kernen. Für die Berechnung wird Gauss-Form vor-

3 G. Römelt, in Proc. Intern. Symp. on Lattice Defects in Semiconductors, Tokyo 1966, Herausg. Hasiguti u. Mrtchell, Unitersity of Tokyo Press, Gordon \& Breach Publ., New York.

4 A. H. Silver u. B. J. Bray, J. Chem. Phys. 32, 288 [1960]. 


\begin{tabular}{|c|c|c|c|c|c|c|}
\hline \multirow{2}{*}{$\begin{array}{l}\text { Umgebung } \\
\text { mit } n{ }^{11} \mathrm{~B} \\
(3-n)^{10} \mathrm{~B} \\
n\end{array}$} & \multirow{2}{*}{$\begin{array}{l}\text { Linien- } \\
\text { zahl }\end{array}$} & \multirow{2}{*}{$\begin{array}{l}N_{n}=1 / \Sigma A= \\
3 \\
{\left[\prod_{(2}\left(2 I_{i}+1\right)\right]^{-1}} \\
i=1\end{array}$} & \multicolumn{2}{|c|}{ natürliches $\mathrm{BN}$} & \multicolumn{2}{|c|}{${ }^{10} \mathrm{BN}\left(91 \%{ }^{10} \mathrm{~B}\right)$} \\
\hline & & & $C_{n}$ & $C_{n} N_{n}$ & $C_{n}$ & $C_{n} N_{n}$ \\
\hline $\begin{array}{l}3 \\
2 \\
1 \\
0\end{array}$ & $\begin{array}{l}10 \\
25 \\
22 \\
19\end{array}$ & $\begin{array}{l}1 / 64 \\
1 / 112 \\
1 / 196 \\
1 / 343\end{array}$ & $\begin{array}{r}\cdot 10^{-2} \\
53,48 \\
37,22 \\
8,63 \\
0,67\end{array}$ & $\begin{array}{l}\cdot 10^{-5} \\
836 \\
332 \\
44 \\
\quad 1,95\end{array}$ & $\begin{array}{r}10^{-2} \\
0,07 \\
2,21 \\
22,36 \\
75,36\end{array}$ & $\begin{array}{c}10^{-5} \\
1,1 \\
19,7 \\
114 \\
220\end{array}$ \\
\hline
\end{tabular}

Tab. 1 .

Zu Tab. 1: Lage und Intensität der Linien.

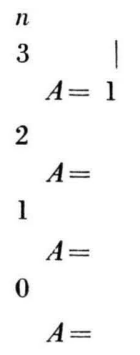

ausgesetzt; für die abgeleitete Absorptionskurve gilt also $f^{\prime}(H) \sim\left(H-H_{0}\right) \exp \left\{-\left(H-H_{0}\right)^{2} / 2 h^{2}\right\}$.

d) Das Gesamtspektrum entsteht durch Addition der Spektren von vier Systemen gemäß der ersten Spalte von Tab. 1. $C_{n}$ ist die statistische Häufigkeit, die aus der Isotopenzusammensetzung folgt.

e) Die relative Intensität $A$ innerhalb jeden Systems und die Linienlage erhält man durch das Aufspalten jeden Energieniveaus in $2 I+1$ Niveaus an jedem der drei Boratome mit dem jeweiligen $I$ und $a$, Tab. 1 unten. Durch den Faktor

$$
N_{n}=1 / \Sigma A=\left[\prod_{i=1}^{3}\left(2 I_{i}+1\right)\right]^{-1}
$$

werden die $A$ auf 1 normiert. Bei der Bildung des Gesamtspektrums ist jede Einzellinie jeden Systems mit dem Faktor $A N_{n} C_{n}$ einzusetzen.
Die Addition der Teilspektren für $2 h=6 \mathrm{Gau}$ ergibt für natürliches BN das Spektrum der Abb. 2, das mit dem experimentellen in Abb. 1 vergleichbar ist, welches bei Zimmertemperatur aufgenommen

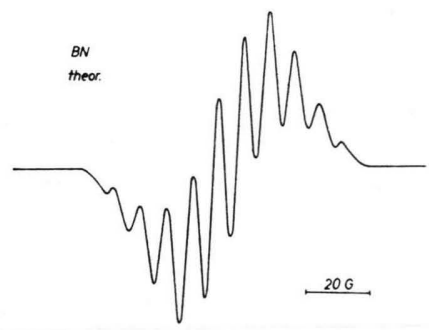

Abb. 2. Berechnetes EPR-Spektrum von BN, nach den Angaben von Tab. 1 mit $2 h=6$ Gauß. $2 h \sqrt{2 \ln 2}=$ Halbwertsbreite der Einzellinie.

wurde. Bessere Übereinstimmung würde im Zentrum mit größerem $2 h$, bei den äußeren Linien mit kleinerer Linienbreite zu erzielen sein. Allerdings ist gerade die mittlere Linie von Sorte zu Sorte verschieden (vgl. Abb. 1 mit Abb. 4 sowie ${ }^{1}$ ).

Die Linienbreite der Einzellinien wächst mit fallender Temperatur und hat bei $77^{\circ} \mathrm{K}$ ein Maximum, so $\mathrm{da} ß$ die Aufspaltung nur noch angedeutet oder völlig verwischt ist. Es tritt dann nur eine einzige Linie auf mit dem Abstand $\Delta H=27 \mathrm{Gauß}$ zwischen den Extrema. Bei einigen Proben ist das Spektrum bei $1,7^{\circ} \mathrm{K}$ wieder besser aufgelöst (Tab. 2).

\begin{tabular}{|c|c|c|c|c|c|}
\hline \multirow{2}{*}{$\begin{array}{c}\text { Tempera- } \\
\text { tur } \\
{ }^{\circ} \mathrm{K}\end{array}$} & \multicolumn{2}{|c|}{ natürliches BN (10 Linien) } & \multirow{2}{*}{$\begin{array}{l}\text { Bor } 5 \\
\\
\Delta H \\
\text { Gauß }\end{array}$} & \multicolumn{2}{|c|}{$\begin{array}{c}{ }^{10} \mathrm{BN}\left(91 \%{ }^{10} \mathrm{~B}\right) \\
(1 \text { Linie })\end{array}$} \\
\hline & $g$ & $\underset{\text { Gauß }}{\text { Einzellinie }}$ & & $g$ & $\begin{array}{c}\Delta H \\
\text { Gauß }\end{array}$ \\
\hline $\begin{array}{r}1,7 \\
77 \\
300 \\
500\end{array}$ & $\begin{array}{l}2,0023 \pm 0,0010 \\
2,0052 \pm 0,0020 * \\
2,0027 \pm 0,0003\end{array}$ & $\begin{array}{r}7 \\
>10 \\
6 \\
6,5 \pm 0,5 \\
6,5\end{array}$ & $\begin{array}{c}15 \\
5 \\
8-10 \\
10-11\end{array}$ & $\begin{array}{l}2,0035 \pm 0,0005 \\
2,0026 \pm 0,0003 \\
2,0029 \pm 0,0003\end{array}$ & $\begin{array}{l}17,5 \pm 1 \\
18 \pm 1 \\
19 \pm 1\end{array}$ \\
\hline
\end{tabular}

* Meßgenauigkeit beeinträchtigt durch überlagerte Linie.

Tab. 2. Meßwerte. 
Im Gegensatz dazu zeigt die am elementaren Bor gefundene einzelne Linie ${ }^{5}$ bei $77^{\circ} \mathrm{K}$ gerade ein $\mathrm{Mi}$ nimum der Halbwertsbreite. Diese Linie ist bei hohen Temperaturen beweglichen Elektronen im Bor zuzuschreiben, während im Bornitrid offensichtlich völlig gebundene Elektronen vorliegen.

Bei angereichertem ${ }^{10} \mathrm{BN}$ hat das stärkste Teilspektrum 19 Linien mit einem Abstand von nur 2,6 Gauß. Daher ergibt die Addition der Teilspektren mit den Intensitäten der Tab. 1 bereits für $2 h=6$ Gauß nur eine einzelne Linie mit $\Delta H=20 \mathrm{Gauß \text {,was }}$ recht gut mit dem Experiment übereinstimmt (Abb. 5 und Tab. 2).

\section{Präparation}

Ausgangsmaterial für die Untersuchungen mit angereichertem ${ }^{10} \mathrm{~B}$ waren kristallines Bor und glasiges $\mathrm{B}_{2} \mathrm{O}_{3}$. Daraus wurde $\mathrm{H}_{3} \mathrm{BO}_{3}$ gewonnen, über $\mathrm{P}_{2} \mathrm{O}_{5}$ im Vakuum bis $200{ }^{\circ} \mathrm{C}$ entwässert und in pulvriges $\mathrm{B}_{2} \mathrm{O}_{3}$ umgewandelt.

Die Herstellung von $\mathrm{BN}$ erfolgte nach ${ }^{6}$ aus a) $\mathrm{B}_{2} \mathrm{O}_{3}$ und Harnstoff, b) $\mathrm{B}_{2} \mathrm{O}_{3}$ und $\mathrm{NH}_{3}$ sowie c) $\mathrm{BBr}_{3}$ und $\mathrm{NH}_{3}$. Am häufigsten und besonders für ${ }^{10} \mathrm{BN}$ wurde a) folgendermaßen durchgeführt: $10 \mathrm{~g} \mathrm{~B}_{2} \mathrm{O}_{3}$ mit $20 \mathrm{~g}$ Harnstoff im geschlossenen Hessischen Tiegel 3 Stunden bei $160{ }^{\circ} \mathrm{C}, 12$ Stunden bei $200^{\circ} \mathrm{C}, 1$ Stunde bei $240{ }^{\circ} \mathrm{C}$ und 1 Stunde unter strömendem Stickstoff bei $1000^{\circ} \mathrm{C}$.

Alle nach a) bis c) gewonnenen Sorten BN lieferten im Rohzustand nach der Herstellung bzw. nach üblichem Waschen und Trocknen gelegentlich einzelne EPRLinien, aber niemals das gesuchte 10-Linien-Spektrum für BN oder die entsprechende Linie für ${ }^{10} \mathrm{BN}$.

\section{Thermisch erzeugte EPR-Zentren. Einfluß der Kristallitgröße}

Der Versuch, die im kommerziellen BN gefundenen EPR-Zentren durch Erhitzen in einer Wolframwendel unter Reinstwasserstoff bis $2000{ }^{\circ} \mathrm{C}$ zu zerstören, mißlang; denn es trat eine geringe Verstärkung des EPR-Signals ein ${ }^{1}$. Dieser Umstand und die im Gegensatz zu handelsüblichem BN nur schwach ausgebildeten Debye-Scherrer-Ringe von selbst hergestelltem BN-Pulver gaben den Anlaß, BN unter Stickstoff zu erhitzen. Geheizt wurde in einem Graphittiegel mit $10 \mathrm{~mm}$ Außen- und $7 \mathrm{~mm}$ Innendurchmesser durch Induktion.

5 D. Geist u. H.-J. Gläser, J. Phys. Chem. Solids 26, 57 [1965]. - W. Klein u. D. Geist, in Vorbereitung.

6 H. J. Becher in G. Brauer, Handbuch der Präparativen Anorganischen Chemie, Ferdinand-Enke-Verlag, Stuttgart 1960.
Das 10-Linien-Spektrum tritt auf, wenn über $1850{ }^{\circ} \mathrm{C}$ erhitzt wird. Bei den kommerziellen Sorten BN, die dieses Spektrum zeigen, dürfte das Erhitzen bereits bei der Herstellung erfolgt sein, besonders beim Sintern.

Das Erhitzen von BN unter Stickstoff ist mit einer Verbesserung der Kristallitstruktur verbunden, und gerade dieser Umstand scheint wesentlich für das Auftreten der EPR zu sein (vgl. IV). So liefert grobkristallines BN (Korngröße bis $100 \mu$ ) das Spektrum der Abb. 1, feinkristallines des gleichen Herstellers überhaupt keine Resonanz (Abb. $3 \mathrm{a}$ ) bzw. erst nach dem Erhitzen auf $1850^{\circ} \mathrm{C}$ (Abb. 3 b).
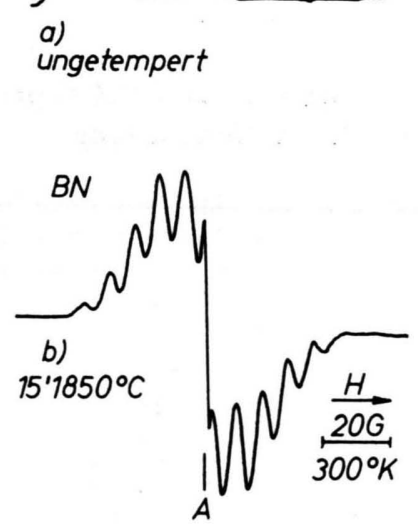

Abb. 3. EPR von feinkristallinem BN. Pulver S I. Modulation $0,875 \mathrm{G} / 250$. a) $240 \mathrm{mg}$, wie vom Hersteller bezogen; b) 120 mg, nach 15 Min. Erhitzen auf $1850^{\circ} \mathrm{C}$ unter $\mathrm{N}_{2}$. Die Einzellinie A dürfte von Kohlenstoff stammen (vgl. V).

Beim selbst hergestellten BN ist trotz gleicher Präparation die Auflösung des 10-Linien-Spektrums unterschiedlich ausgeprägt. Sie ist gut, wenn beim Erhitzen über $1500{ }^{\circ} \mathrm{C}$ wenig Substanz verdampft (vermutlich $\mathrm{B}_{2} \mathrm{O}_{3}$ ), schlecht nach starkem Verdampfen. In letzteren Fällen ist offensichtlich die Umsetzung bzw. Kristallbildung schlecht verlaufen.

Bemerkenswert ist in diesem Zusammenhang das Ergebnis der Röntgen-Beugungsversuche von THOmas et al. ${ }^{7}$, daß feinkristallines und relativ ungeordnetes BN mit schwachen Ringen durch Erhitzen bei Anwesenheit von $\mathrm{B}_{2} \mathrm{O}_{3}$ besser kristallisiert und gut ausgeprägte Ringe zeigt. Die Umwandlung ist bei $1850{ }^{\circ} \mathrm{C}$ vollständig, also gerade bei der Tempera-

\footnotetext{
7 J. Thomas jr., N. E. Weston u. T. E. O'Connor, J. Am. Chem. Soc. 84, 4619 [1963].
} 
tur, die zum Entstehen der EPR-wirksamen Zentren erforderlich ist. Ohne Mitwirkung von $\mathrm{B}_{2} \mathrm{O}_{3}$ bleibt der ungeordnete Zustand selbst bei $2000{ }^{\circ} \mathrm{C}$ erhalten. Bei allen untersuchten Sorten BN dürfte der Gehalt an $\mathrm{B}_{2} \mathrm{O}_{3}$ groß genug sein, um eine Kristallisation nach ${ }^{7}$ zu fördern. Bei dem nach Verfahren II a) hergestellten ist $\mathrm{B}_{2} \mathrm{O}_{3}$ die Ausgangssubstanz. Für käufliches gesintertes BN wird ein Sauerstoffgehalt von einigen Prozent angegeben, aber auch pulverförmiges enthält bis zu $0,5 \%$. Abgesehen von der mitunter schlechten Auflösung ist das 10-LinienSpektrum auf jeden Fall nach Erhitzen auf über $1850{ }^{\circ} \mathrm{C}$ aufgetreten, in angereichertem ${ }^{10} \mathrm{BN}$ mit 91\% ${ }^{10} \mathrm{~B}$ die entsprechende Linie mit $19 \mathrm{Gau} ß \mathrm{Ab}$ stand zwischen den Extrema.

\section{Erzeugung von EPR-Zentren durch Bestrahlung}

Die EPR-Zentren, die das 10-Linien-Spektrum von natürlichem BN bzw. die breite Einzellinie von ${ }^{10} \mathrm{BN}$ verursachen, kann man auch durch Bestrahlen mit ultra-

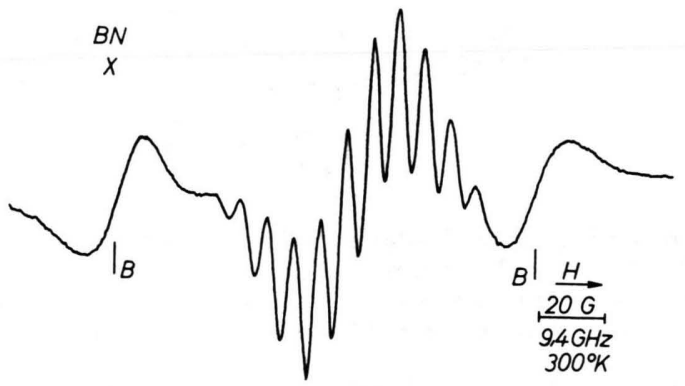

Abb. 4. EPR von bestrahltem BN. $220 \mathrm{mg}$ gesintertes BN CV nach 30 Min. Bestrahlung mit Mo-RöNtgen-Strahlen mit $60 \mathrm{kV}$, $12 \mathrm{~mA}$. Magnetfeld senkrecht zur Stabachse, also beste Auflösung des Spektrums. Die zwei Linien (B, B) neben dem 10-Linien-Spektrum gehören zu einem instabilen 4-LinienSpektrum, das ebenfalls erst nach dem Bestrahlen auftritt ${ }^{3,8}$. Modulation 0,44 G/400.

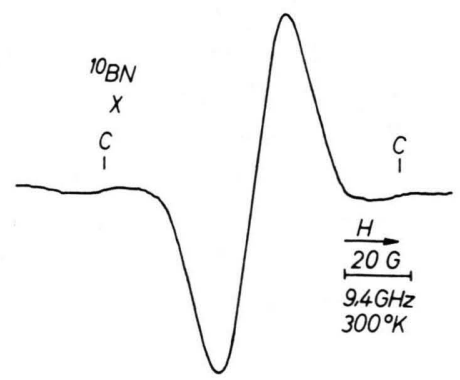

Abb. 5. EPR von ${ }^{10} \mathrm{BN}\left(91 \%{ }^{10} \mathrm{~B}\right) .145 \mathrm{mg}$ gesintertes ${ }^{10} \mathrm{BN}$ W2 nach 15 Min. Bestrahlung mit Cu-Röntgen-Strahlen mit $45 \mathrm{kV}$, $14 \mathrm{~mA}$. Die beiden Nebenlinien (C, C) gehören zu einem instabilen 7-Linien-Spektrum ${ }^{3,8}$. Modulation 0,35 G/250. violetten, RöNTGEN- oder $\gamma$-Strahlen erzeugen (Abb. 4 und 5). Dabei entstehen auch noch andere Zentren, die ebenfalls EPR-wirksam sind und offensichtlich nur mit einem Bor-Kern in Wechselwirkung stehen ${ }^{3,8}{ }^{8}$. Diese Zentren sind nicht stabil, im Gegensatz zu denen, die bei Neutronenbestrahlung gebildet werden, bisher aber nicht gedeutet werden können ${ }^{8}$.

Bestrahlt wurde bei Zimmertemperatur mit den ungefilterten RöNTGen-Strahlen von Cu- und Mo-Röhren mit 25 bis $60 \mathrm{kV}$ Anregungsspannung, dem ungefilterten Licht einer Quecksilberlampe Osram $\mathrm{Hg} 3$ sowie den $\gamma$-Strahlen einer ${ }^{60} \mathrm{Co}$-Quelle. Die EPR-Spektren sind gleich, obwohl die Energien der Photonen sich erheblich unterscheiden $(25-60 \mathrm{keV}$, einige $\mathrm{eV}$ bzw. etwa $1 \mathrm{MeV} ; k T$ beim Erhitzen auf $1850{ }^{\circ} \mathrm{C}$ entspricht $0,2 \mathrm{eV}$; für den Bandabstand von hexagonalem $\mathrm{BN}$ werden Werte von $3,6-4,6 \mathrm{eV}^{\text {genannt }}{ }^{9}$.

Die Bestrahlung ergänzt die beim Erhitzen gewonnenen Ergebnisse und ermöglicht insbesondere auch Aussagen darüber, wie groß die Spindichte werden kann, die das 10-Linien-Spektrum verursacht: Es zeigt sich, daß die Dichte der Spinzentren durch langes Bestrahlen nicht beliebig erhöht werden kann; sie strebt vielmehr einem Grenzwert zu, der nicht höher als $8 \cdot 10^{17}$ Spins/g liegt oder anschaulicher: Von den $2,4 \cdot 10^{22}$ Ringen/g BN (= Boratomen /g BN) hat höchstens jeder $3 \cdot 10^{4}$-te einen EPRwirksamen Spin. Die Spindichte wurde durch Vergleich mit $\mathrm{CuSO}_{4}$ bestimmt.

Die Grenze ist dann erreicht, wenn Erzeugung und Rekombination der Zentren gleich groß werden. Allerdings ist die Frage nach der Stabilität dieser Zentren bisher nicht eindeutig zu beantworten: Einerseits existieren Proben, die schon seit zwei Jahren ein gleich starkes Spektrum zeigen, andererseits fällt nach dem Bestrahlen mit der Zeit die Intensität anderer Proben. Es entsteht der Eindruck, daß eine gewisse Dichte von Zentren über lange Zeit bestehen bleiben kann, während darüber hinausgehende langsam verschwinden. Eine Beteiligung von Verunreinigungen an diesen Prozessen ist nicht ausgeschlossen, bisher aber nicht nachgewiesen.

Auch beim bestrahlten BN zeigt sich ein Einfluß der Kristallitgröße auf die EPR, der die Angaben unter III bestätigt: Bei feinkristallinem Pulver eigener und fremder Herstellung ist das 10-Linien-Spektrum durch Bestrahlen nur sehr schwach zu gewinnen im Gegensatz zu grobkristallinem und insbesondere gesintertem BN. Nach vorherigem Erhitzen ist auch das Bestrahlen wirksamer.

8 D. Geist, G. Römelt u. W. Klein, Symposium on Lattice Defects in Semiconductors, Tokyo 1966.

9 N. A. Goryunova, The Chemistry of Diamon-like Semiconductors, Chapman and Hall, London 1965. 


\section{EPR von C-dotiertem BN}

Da eine Beteiligung von Fremdstoffen an den EPR-Zentren bisher nicht auszuschließen ist, wurde versucht, durch Beimischung von Fremdstoffen das EPR-Spektrum zu beeinflussen. $100 \mathrm{mg} \mathrm{CrO}_{3}$ und $\mathrm{MnO}_{2}$ bei den Ausgangsstoffen erwiesen sich als unwirksam, obgleich das Roh-BN nach dem Erhitzen auf $1000{ }^{\circ} \mathrm{C}$ noch gefärbt war. Nach dem Erhitzen auf $1850^{\circ} \mathrm{C}$ war das $\mathrm{BN}$ wieder weiß und das 10 Linien-Spektrum gut aufgelöst.

Auch Kohlenstoff, der in Form von Zucker den Ausgangsstoffen oder dem Roh-BN vor dem Erhitzen auf $1850{ }^{\circ} \mathrm{C}$ zugefügt wurde, veränderte nicht das 10-Linien-Spektrum, lieferte aber eine zusätzliche schmale Linie bei $g=2$. Auch die zusätzliche Linie in Abb. 3 b dürfte auf Kohlenstoff zurückzuführen sein, der in technischem BN als Verunreinigung recht häufig vertreten ist.

In diesem Zusammenhang sei auch auf die Beobachtung hingewiesen, daß das 10-Linien-Spektrum von selbst hergestelltem $\mathrm{BN}$ dann besonders gut aufgelöst war, wenn das Roh-BN starke grünliche Lumineszenz zeigte. (Das waren die Sorten mit geringem Verdampfen, vgl. II.) Als Ursache der Lumineszenz von $\mathrm{BN}$ ist seit langem Kohlenstoff bekannt ${ }^{10}$. Auch alle Sorten gekauften Bornitrids zeigen Lumineszenz, auch diejenigen, die kein EPR-Spektrum liefern.

\section{Diskussion}

Ähnlich dem Graphit (Raumgruppe C6/mmc; $\left.\mathrm{D}_{6 \mathrm{~h}}{ }^{4}\right)$ hat Bornitrid $\left(\mathrm{C} \overline{\mathrm{m}} 2 ; \mathrm{D}_{\mathbf{3}}{ }^{12}\right)$ ein Schichtengitter mit dem Schichtabstand 3,35 § und dem Abstand $\mathrm{B}-\mathrm{N}=1,45 \AA$. Im Gegensatz zum Graphit sind beim BN sämtliche Atome der Schichten genau übereinander angeordnet ${ }^{9}$. Wegen des relativ großen Schichtabstandes dürften bei der Wechselwirkung in erster Näherung nur Atome der gleichen Schicht beteiligt sein.

Da offensichtlich jeweils drei Boratome von der Hyperfeinwechselwirkung erfaßt werden, müssen die EPR-Zentren entweder auf Stickstoffplätzen oder im Zentrum der Ringe sitzen.

10 F. WöHLER, Liebigs Ann. Chem. 74, 71 [1850].
Für ein nicht abgesättigtes Elektron im Zentrum eines $\mathrm{B}_{3} \mathrm{~N}_{3}$-Ringes mit der gleichen Aufenthaltswahrscheinlichkeit $|\psi(0)|^{2}$ an den je drei umgebenden Bor- und Stickstoffkernen müßte jede der 10 Einzellinien wegen des Kernspins von ${ }^{14} \mathrm{~N}(I=1, \mu / I=0,4)$ nochmals im Verhältnis $1: 3: 6: 7: 6: 3: 1$ in 7 Linien mit dem Abstand 1,7 Gauß aufspalten. Ein EPRZentrum am Ort eines Stickstoffatoms hat nur drei Boratome als nächste Nachbarn und würde wegen der 6 übernächsten Nachbarn (N) mit anderem $|\psi(0)|^{2} \mathrm{zu}$ einer anderen Aufspaltung führen. Welche der genannten Möglichkeiten tatsächlich zutrifft, ist besonders wegen der großen Linienbreite noch nicht zu entscheiden.

Im Gegensatz zu elementarem Bor, wo die EPR vermutlich durch bewegliche Elektronen verursacht wird, rührt die EPR des Bornitrids von gänzlich gebundenen Elektronen her.

Die Struktur des BN und das Aussehen des EPRSpektrums legen einen Vergleich mit den freien aromatischen Radikalen nahe. Dabei zeigen sich folgende Unterschiede:

1. Bei den freien Radikalen ist das nicht abgesättigte Elektron in Wechselwirkung mit allen Protonen usw. des Moleküls, auch solchen an Nebenringen und Seitenketten; die Kohlenstoffatome, die den Ring bilden, haben keinen Kernspin. Beim BN ist das Elektron auf drei Boratome beschränkt.

2. Im unverdünnten Kristall sind die Spindichten bei freien Radikalen allgemein so hoch, daß Wechselwirkungen zwischen den Zentren zum Verschwinden der Hyperfeinaufspaltung führen und nur eine einzelne schmale Linie übrigbleibt, z. B. beim DPPH. Im $\mathrm{BN}$ ist dagegen die Dichte der EPR-wirksamen Zentren so klein, daß keine Austauschverschmälerung auftritt. Durch Bestrahlung entsteht nicht mehr als 1 Spin auf $3 \cdot 10^{4}$ Boratome, was möglicherweise durch Beteiligung von Fremdatomen verursacht wird.

Herrn Professor Dr. J. JaumanN, II. Physikalisches Institut der Universität zu Köln, danke ich für die in seinem Institut großzügig gewährten Arbeitsmöglichkeiten. Herrn Professor Dr. D. GeIsT danke ich für die Anregung zu diesen Untersuchungen und die Möglichkeit zu ihrer Durchführung in seinem Institut, insbesondere mit Meßgeräten und Materialien, die von seiten der Deutschen Forschungsgemeinschaft zur Verfügung standen. 